\title{
Red meat consumption leads to a microbiota-dependent risk of cardiovascular disease
}

The gut microbiota of individuals who frequently eat red meat contributes to an increased risk of atherosclerosis, a study published in Nature Medicine shows.

The link between a diet rich in red meat and the development of cardiovascular disease-even when levels of saturated fat and cholesterol are controlled for-is well-established. Koeth et al. investigated whether the intestinal microbiota influences cardiovascular risk and determined the part played by L-carnitine, a nutrient abundant in red meat.

The study participants consisted of 51 omnivores and 26 vegetarians or vegans, a subset of which was asked to ingest $180 \mathrm{mg}$ of L-carnitine (in the form of an 8-oz sirloin steak) and/or $250 \mathrm{mg}$ of isotope-labeled $\mathrm{L}$-carnitine. Tracing of the isotope in plasma and urine showed that trimethylamine- $N$ oxide (TMAO), a compound known to be associated with cardiovascular risk, was highly produced in meat-eaters but barely

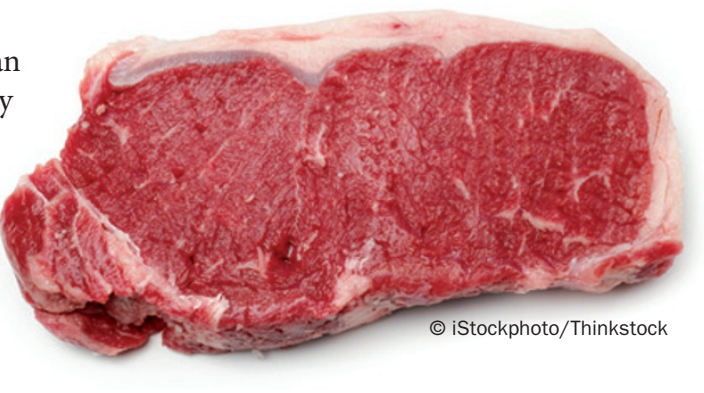

detectable in vegetarians and vegans. The role of the gut microbiota was examined by providing a 1-week course of broadspectrum antibiotics, followed by another L-carnitine challenge. After this treatment, TMAO levels were undetectable, which suggests that intestinal microbiota are necessary for the metabolism of TMAO from dietary L-carnitine.

The gut microbial taxa of individuals were found to depend on their chronic dietary habits (omnivores vs vegetarian or vegans), and TMAO synthesis seems to be associated with certain bacterial genera.
Furthermore, Koeth et al. measured the blood L-carnitine levels in 2,595 persons undergoing elective cardiac evaluation. High plasma levels of L-carnitine was associated with a raised risk of cardiovascular-related adverse events but only in combination with elevated TMAO levels, highlighting a role for TMAO rather than $\mathrm{L}$-carnitine in the development of cardiovascular diseases.

Finally, experiments in mice indicated that TMAO promote atherosclerosis by suppressing the transfer of cholesterol from peripheral tissues to the liver. The researchers conclude that a high intake of L-carnitine could be associated with potential health risks.

Elisabeth Kugelberg

Original article Koeth, R. A. et al. Intestinal microbiota metabolism of L-carnitine, a nutrient in red meat, promotes atherosclerosis. Nat. Med. doi:10.1038/nm.3145 